\title{
Influence of Orientation Mismatch on Charge Transport Across Grain Boundaries in Tri-isopropylsilylethynyl (TIPS) Pentacene Thin Films
}

\author{
Florian Steiner, ${ }^{1}$ Carl Poelking, ${ }^{2}$ Dorotha Niedzialek, ${ }^{1}$ Denis Andrienko, ${ }^{2}$ and Jenny Nelson ${ }^{1}$ \\ ${ }^{1}$ Department of Physics and Centre for Plastic Electronics, Imperial College London, London SW7 2AZ \\ ${ }^{2}$ Planck Institute for Polymer Research, Ackermannweg 10, 55128 Mainz
}

\begin{abstract}
We present a multi-scale model for grain boundaries quantifying physical characteristics such as inter-granular energy barriers and electronic coupling elements on a molecular scale and simulate their effect on charge transport. We choose thin films of tri-isopropylsilylethynyl pentacene (TIPS-P) as a model system that is representative of technologically relevant crystalline organic semiconductors. We use atomistic molecular dynamics, with a force-field specific for TIPS-P, to generate and equilibrate polycrystalline thin films. The energy landscape is obtained by calculating contributions from electrostatic interactions and polarization. Subsequently, charge transport is simulated using a kinetic Monte-Carlo algorithm. Two-grain systems with varied mutual orientation are studied. We find relatively little effect of long grain boundaries due to the presence of low impedence pathways. However, effects could be more pronounced for systems with limited inter-grain contact areas. Furthermore, we present a lattice model to generalize the model for small molecular systems. Depending on molecular architecture and packing, grain boundaries can result in interfacial energy barriers, traps or a combination of both. Consequently, this study provides a new, microscopic perspective on how grain boundaries limit charge transport in crystalline molecular thin films.
\end{abstract}

\section{Introduction}

Solution-processing of small-molecule crystalline semiconductor films generally leads to polycrystalline films containing crystallites with sizes of tens of $\mathrm{nm}$ to several $\mu \mathrm{m}$ separated by grain boundaries and amorphous interphases ${ }^{13,16,17,30}$. The presence of grain boundaries reduces charge carrier mobilities compared to single-crystal films deteriorating device characteristics such as the switching speed of field-effect transistors (FET). Although much effort has been spent on increasing crystal sizes ${ }^{8-10,34}$, device reproducibility is poor since few grain boundaries are sufficient to impede device performance severely (lowering mobilities by several orders of magnitude) ${ }^{12,29}$.

Charge transport in FETs can be considered quasi two-dimensional along a channel parallel to the gate electrode ${ }^{23}$. Within single-crystal grains, charge-carrier mobilities can be highly orientation dependent with mobility anisotropies larger than $10^{20,25}$. At grain boundaries, Kelvin probe force microscopy exhibits surface potential wells suggesting trap states ${ }^{28}$. This observation is in agreement with conductive probe atomic force microscopy results revealing several orders of magnitude higher resistances for grain boundaries ${ }^{17}$. Rivnay and co-workers suggested that the impact of grain boundaries on charge transport is dependent on the crystal packing structure ${ }^{29}$. His qualitative picture implied that Herringbone packed materials (e.g. pentacene) suffer less from grain boundaries than materials in brickwork structures (e.g. TIPS-P). The larger the mutual angle between grains, the more severe would be the effect of grain boundaries due to reduced electronic coupling of molecules at the interface. This angular dependence is supported by a study of Lee et al. obtaining lower mobilities for larger mutual angles between grains for 5,11-bis(triethylsilylethynyl)anthradithiophene (TES-ADT) ${ }^{21}$. In both studies, the general picture is that grain boundaries in small molecular systems are sharp and abrupt. In contrast, Wong and co-workers claim that grain boundaries between TIPS-P grains consist of small crystals that are somehow connected to each other and grain boundaries are therefore expected to be small and surrounded by voids ${ }^{37}$.

Kelvin probe measurements of the surface potential on polycrystalline silicon have lead to drift-diffusion models with trap states at the grain interface $\mathrm{e}^{2,33}$. The method was later adopted for other polycrystalline inorganic and organic semiconductors ${ }^{14,22}$. The approach suggests that in organic FETs high charge concentrations in the channel lead to back-to-back Schottky barriers forming around grain boundaries. Despite allowing for an effective-medium interpretation of device characteristics, drift-diffusion-based models are restricted to one dimension and fitting to macroscopic parameters (e.g. trap concentration, width of grain boundaries). Moreover, the formation of back-toback Schottky barriers requires high doping concentrations at the grain boundary which is questionable for organic semiconductors. On the other hand, donor-acceptor interfaces have been studied on a molecular scale to understand exciton dissociation in organic solar cells ${ }^{6,7,27}$. Cornil and co-workers could show that the energy landscape at the 
interface between two different materials depends on the mutual orientation of the molecules ${ }^{6}$. Moreover, Poelking et al. suggested that long-range interactions are crucial to understand level alignment at donor-acceptor interfaces ${ }^{27}$.

In this study, we address the question whether grain boundaries cause disruptions to the energy landscape and if so, whether barriers or traps are formed, or if grain boundaries only present regions of poor electronic coupling. We use multi-scale modeling to understand the microscopic properties of grain boundaries in small-molecular organic thin films. We use atomistic molecular dynamics to simulate grain boundaries with varied mutual grain orientations $\vartheta$, and explore the dependence on $\vartheta$ of hole transport across grains using a kinetic Monte-Carlo hopping model. The energetic landscape across the grain boundary is modelled by calculating electrostatic and inductive contributions to site energies. We find that the grain boundary in TIPS-P introduces a barrier to charge transport, but that the impact on transport across is weak and independent of $\vartheta$, unless the contact area between grains is small. Extensions to the approach using a lattice description show that in general grain boundaries may lead to interfacial energetic barriers, energetic traps, or both, depending on the molecular architecture, packing and the charge-carrier type (electron or hole).

\section{Methods}

a. i) Generating Molecular Assemblies Generating realistic molecular assemblies of relevant size for charge transport simulations is challenging due to computational constraints ${ }^{35}$. In this case the challenge is simplified by the tendency of TIPS-P to organise with the $\mathbf{a}-\mathbf{b}$ plane parallel to the substrate (e.g. on silicon, glass and amorphous carbon see reference ${ }^{5}$ ). In this plane, TIPS-P exhibits good two-dimensional transport properties due to high transfer integrals in the $\mathbf{a}$ and $\mathbf{a}-\mathbf{b}$ directions, constraining charge transport to a more or less two-dimensional plane since coupling in the $\mathbf{c}$ direction is very weak ${ }^{36}$. In this study we take two approaches to generate representative systems. First, we use atomistic molecular dynamics (MD) to model the arrangement of TIPS molecules around grain boundaries in a two-crystallite system. Later, we complement the model with a coarse-grained approach where abrupt boundaries between grains are simulated on a lattice populated with anisotropic quadrupolar particles.

b. Atomistic Models To generate polycrystalline assemblies, we first prepare two grains with a mutual orientation $\vartheta$ (the relative orientation of corresponding a vectors). $\vartheta$ is varied between 0 (parallel grains) and 90 degrees (vertical grains) in steps of 22.5 degrees. Initially, both grains are built using the crystal structure of reference ${ }^{1}$ and are chosen to be separate by few nanometers (around $1 \mathrm{~nm}$ ). This distance is close enough for TIPS-P molecules of different grains to interact, and far enough to allow for orientational and positional equilibration at the grain-interface during the MD simulation. A molecule-specific force-field is developed using the OPLS reference data base ${ }^{15}$. Force-field parameters between silicon atoms (in the TIPS-P side-chains) and surrounding atoms are either taken from Guilbert et al. ${ }^{11}$ or fitted comparing molecular mechanics calculations with a molecular structure derived from hybrid density functional theory calculations (B3LYP/6-311g**). The fitting process of force-field parameters was repeated iteratively until bond length differences between the optimised quantum-chemical geometry and the structure calculated with molecular mechanics were below $0.001 \AA$. The complete force-field for TIPS-P can be found in Appendix A.

The initial two-grain structures are relaxed in an NPT ensemble. Pressure is applied with an anisotropic barostat (Berendsen) parallel to the two-dimensional system. The equilibration is performed under periodic boundary conditions. Note that the out-of-plane cell dimension of the $2 \mathrm{D}$ system is $5 \mathrm{~nm}$, preventing interactions along this direction. MD simulations are run for $10 \mathrm{~ns}$. The resulting molecular assemblies are quadratic with side-lengths of about $40 \mathrm{~nm}$ and contain around 2,600 molecules. Figure 1a illustrates an equilibrated structure with a mutual angle $\vartheta$ of 67.5 degrees. Grains maintain their general alignment during equilibration, i.e., the grain orientation is still visible in the final structures. Exclusively at the interface between grains, molecules displace and can adopt completely different orientations. Parallel grains ( $\vartheta$ equals 0 degrees) crystallise and hence no grain boundary is observable. For the charge transport calculations, snapshots are extracted after $6,7,8,9$, and 10 ns for each configuration angle $\vartheta$.

c. Lattice Models To generate the lattice morphologies, several (approximately) periodically matched sublattices assembled from unit cells with varying orientations are superimposed and seeded independently, where each sublattice corresponds to a given in-plane crystallite alignment. The unit cell of the crystals has a bcc-structure (lattice constant $c=0.7 \mathrm{~nm})$ with a monomolecular basis of quadrupolar $\left(Q_{20}=-10 \mathrm{au}\right)$ lattice sites. The individual grains are subsequently grown probabilistically until the simulation cell of $75 \times 75 \times 5 \mathrm{~nm}^{3}$ is densely populated based on a site-site exclusion potential $V(i, j)=\infty$ if $r_{i j} \leq r_{c}$, else 0 , with particle indices $i, j$ and cutoff $r_{c}=\sqrt{3} / 2 c$.

d. ii) Site-Energy Calculations Site energies $E$ are computed considering electrostatics only or electrostatics and polarization following reference ${ }^{31}$. Molecular fields are parametrized via a a distributed multipole analysis built on atomic expansion sites. The molecular field response is described within the Thole point-dipole model, which damps 
dipole-dipole at short interaction distances to correct for unphysical polarization divergences. The established way of calculating site energies from electrostatics and polarization is by assuming an interaction cutoff, beyond which no interactions between multipoles are considered, to reduce computational effort. For comparison, we also include a long-range polarized embedding technique which rigorously accounts for long-range interactions crucial to describe out-of-plane level alignment ${ }^{27}$. Within the lattice model, the same approach is applied, using, however a single-site electrostatic representation of the molecular building blocks.

e. iii) Transport Simulation Charge transport is simulated for two-grain MD structures with a kinetic Monte-Carlo approach using ToFeT ${ }^{19}$. Hopping rates are computed from Marcus theory. An electric field of $10^{7} \mathrm{~V} / \mathrm{m}$ is applied perpendicularly to the grain boundary, here corresponding to the field-independent regime of the charge-carrier mobility. Hole mobilities are quantified by the average speed of charge carriers calculated from collection times. Simulations are run in the limit of low carrier concentrations and with regenerative contacts of width $2 \mathrm{~nm}$ at each end. Previously, we calculated the inner-sphere reorganisation energy of TIPS-P to be 138 meV ${ }^{36}$. The external contribution to the reorganisation energy is hard to estimate ${ }^{24}$. While temperature-dependent mobility measurements advocated band-like transport in TIPS-P ${ }^{32}$, Hall-effect measurements revealed charge-carrier localisation and hopping ${ }^{4}$. Therefore, we assume relatively small reorganisation energies $\lambda$ of $200 \mathrm{meV}$ for TIPS-P. For completeness, we also consider $\lambda=0.5 \mathrm{eV}$.

Charge-transfer integrals $J$ are computed with the Molecular Orbital Overlap (MOO) method ${ }^{18}$. MOO allows fast evaluations of electronic coupling elements compared to other approaches such as the projective method $^{3}$, since orbitals are calculated with the semi-empirical differential overlap functional ZINDO and computation of molecular pairs can be omitted. The increased computation speed is of great advantage for systems with several thousand molecules as in this study. The ZINDO parametrization excludes heavy elements such as silicon. Therefore, we drop parts of the side-chain structure of TIPS-P for transfer integral calculations by substituting the silicon end group with hydrogen (see Appendix B). We belief that this is a good approximation for transfer integral calculations given that the $\pi$-electron system of TIPS-P is located on the pentacene core. Appendix B illustrates that transfer integrals from the projective method (full TIPS-P geometry) and MOO (reduced geometry with hydrogen instead of silicon) are very similar and the two-dimensional transport behaviour is preserved. Considering the ideal crystal structure of TIPS-P in reference ${ }^{1}$, the two dominating coupling elements in the $\mathbf{a}$ and $\mathbf{a}-\mathbf{b}$ direction amount to $85.7 \mathrm{meV}$ and $70.0 \mathrm{meV}$, respectively (calculated with MOO).

\section{Dynamics and Energetics in Two-Grain Systems of TIPS-P with Orientational Mismatch}

f. i) Charge Transfer Parameters Figure 1 summarises the findings for a model structure from atomistic MD with a mutual angle of 67.5 degrees. Apart from the case of $\vartheta=0$ degrees, all structures show grain boundaries and an associated energy barrier between the grains. Note that the barrier height is non-uniform (see Figure 1b). Peak barrier heights increase with $\vartheta$. The first two graphs of Figure 1c are projections of site-energies onto the axis vertical to the grain boundary. Compared to electrostatics-only calculations, polarization slightly reduces energetic disorder and homogeneously stabilizes energy levels by only around $0.2 \mathrm{eV}$ as a consequence of the membrane- rather than bulk-like setup. The average energy barrier height is, however, maintained.

The bottom graph in Figure 1c shows transfer integrals projected onto the axis perpendicular to the grain boundary. Each transfer integral is assigned to the center of mass of the charge-transfer pair. Within the grains, two bands at around $10^{-2} \mathrm{meV}$ and $10^{-5} \mathrm{meV}$ can be observed. The former relates to the strong electronic coupling along the $\mathbf{a}$ and $\mathbf{a}-\mathbf{b}$ directions, whereas the latter reflects transfer along the $\mathbf{b}$ direction. The $\mathbf{a}$ and $\mathbf{a}-\mathbf{b}$ transfer integrals are slightly smaller than in the ideal crystal structure of TIPS-P due to thermal disorder introduced by molecular dynamics. At the grain boundary, the higher band drifts towards lower transfer integrals while the lower band vanishes. The higher the mutual angle $\vartheta$, the larger is the reduction in electronic coupling at the grain boundary (see Appendix E)

The results of our microscopic calculations are in line with experimental findings. At grain boundaries we observe barriers for hole transport which would appear as potential wells in Kelvin probe measurements. Simulated and experimental potential well depths are both on the order of $100 \mathrm{meV}$. Moreover, we find that the inter-granular trap depth is non-uniform. Similar to the picture developed by Rivnay et al., the electronic coupling decreases with increasing mutual angles $\vartheta$. 
a)

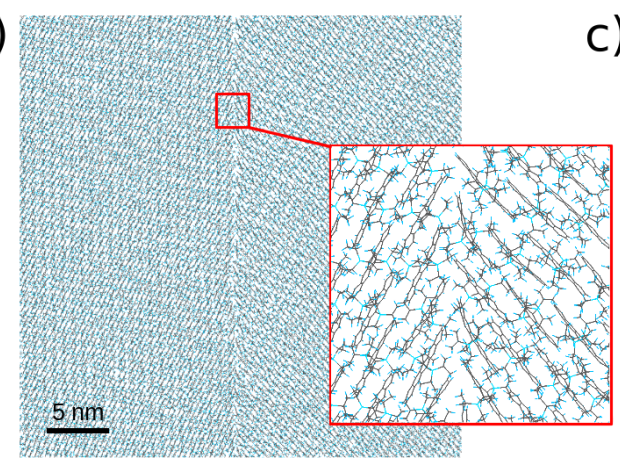

b)

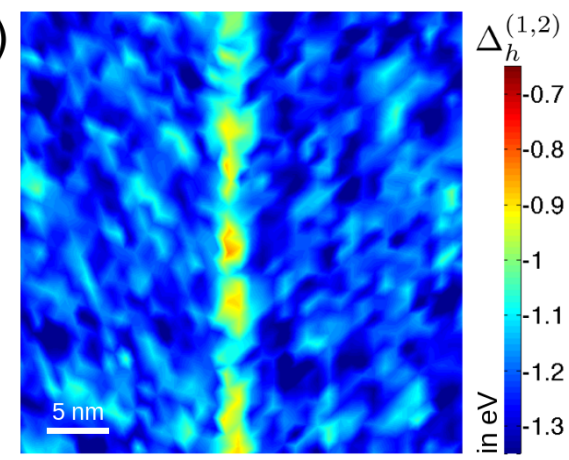

c)

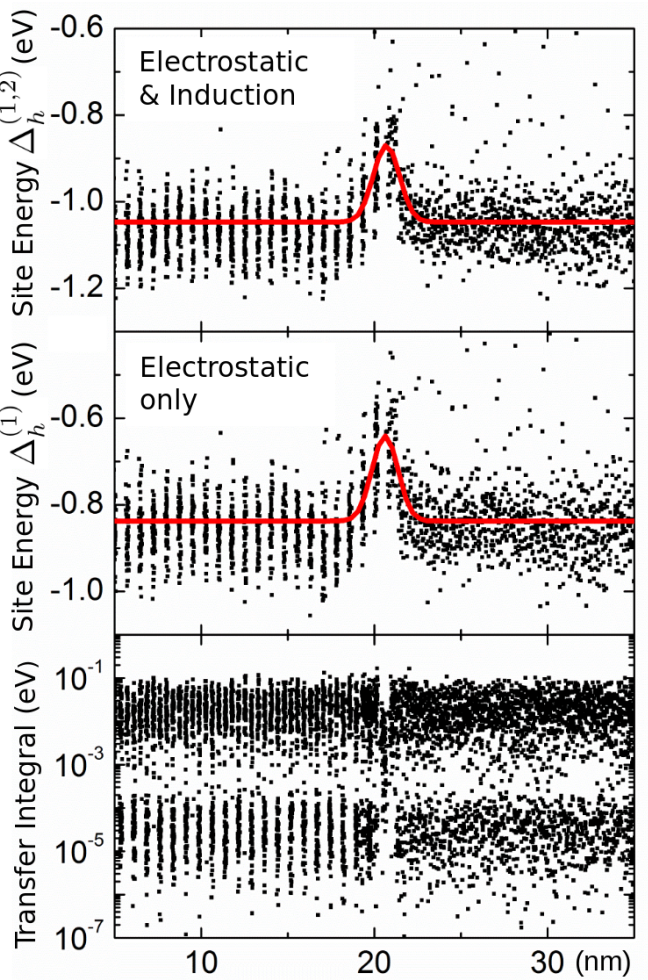

FIG. 1: Results of molecular dynamics simulations and computation of site energies and transfer integrals for a grain/grain angle $\vartheta$ of 67 degrees. a) Equilibrated molecular dynamics structure after $10 \mathrm{~ns}$, b) energy landscape for cutoff calculations including electrostatics and polarization and c) projection of site energies (top) and (middle) and transfer integrals (bottom) onto the axis vertical to the grain boundary.

g. ii) Charge Transport Charge transport is simulated on i) the complete $400 \mathrm{x} 400 \AA^{2}$ atomistic MD structures and on ii) $40 \times 400 \AA^{2}$ stripes confining charge carriers to small sections of the grain boundary. Figure 2 summarises the results for the complete $400 \times 400 \AA^{2}$ molecular assemblies. Mobilities are simulated both with $(\mu)$ and without $\left(\mu_{E=\text { const }}\right)$ energetic disorder. Furthermore, mobilities of the ideal crystal structure of TIPS-P $\left(\mu_{X t a l}\right)$ are calculated through direct solution of the Master equation. Here, $\mu_{X t a l}$ excludes disorder in both site energies and electronic couplings, such that this mobility depends solely on the crystal orientation of the two grains ${ }^{36}$. As described above, we consider for each angle $\vartheta 5$ different molecular assemblies (molecular dynamics snapshots) separated by $1 \mathrm{~ns}$ in time. Mobility ratios are calculated individually for each structure. Subsequently, the average ratio is computed.

Figure 2a reflects the influence of the energy landscape on hole mobilities, which is approximately constant for all angles. For each $\vartheta$, electrostatics-only calculations exhibit on average lower mobilities than simulations including polarization. This should be attributed to the decrease in energetic disorder upon polarization (see the previous section). Comparing mobilities at constant site energies and in the ideal crystal structure allows an estimation of the effect of disorder in electronic couplings (see Figure 2b). Just as for the energy landscape, there is no obvious $\vartheta$-dependence of the effect of disorder in electronic couplings on charge transport. Disorder in transfer integrals, however, seems to have a stronger effect on the absolute mobilities than disorder in site energies: The average ratio $\mu_{E=\text { const }} / \mu$ is around 4 , whereas $\mu_{X t a l} / \mu_{E=\text { const }}$ is exceeds 50 .

Finally, the absolute mobility reduction compared to the ideal crystal structure without disorder in electronic couplings and site energies is discussed in Figure 2c. For 0 degrees, the average mobility ratio $\mu_{X t a l} / \mu$ is slightly lower than for larger $\vartheta$. Again, no clear trend for increasing $\vartheta$ is observable. Thus, the large, $400 \times 400 \AA^{2}$ morphologies appear unable to explain the experimentally observed mobility reductions due to grain boundaries. As we, however, discussed in the previous section, barrier height and transfer integrals are non-uniform at the grain/grain interface. We will show below that charge carriers are as a result likely to find 'sweet spots' along the boundary, characterized by a smaller energy barriers and strong electronic couplings.

The way the situation changes when confining charge transport to $40 \AA$ wide boundary stripes is summarized 


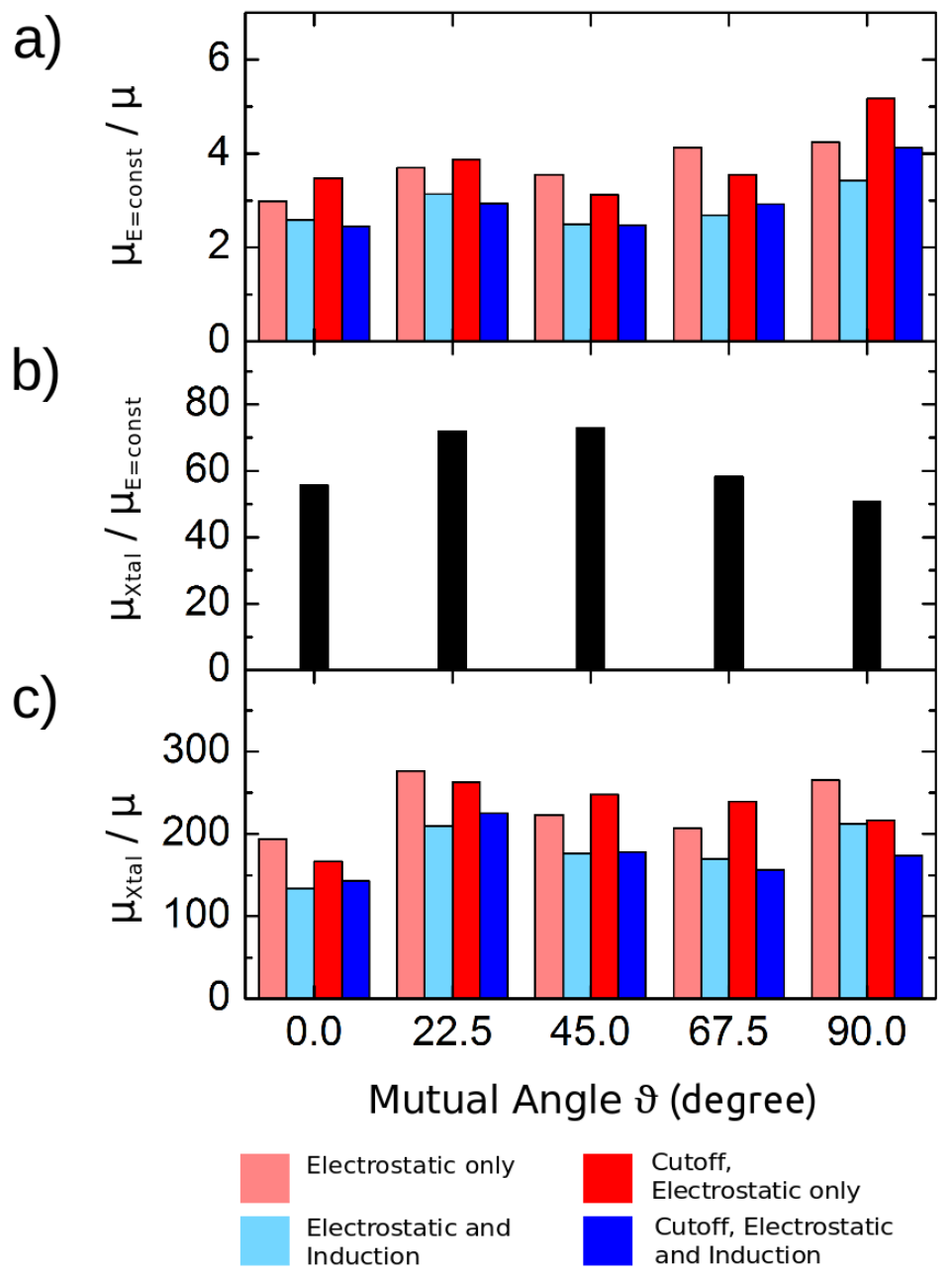

FIG. 2: Results of charge transport simulations of large, $40 \times 40 \mathrm{~nm}^{2}$ structures with one grain boundary and varied mutual crystal orientations. $\mu_{X \text { tal }}$ refers to the mobility of the ideal crystal structure, $\mu_{E=\text { const }}$ relates to the mobility of the equilibrated molecular dynamics structure without energetic disorder and $\mu$ is the mobility of the equilibrated molecular dynamics structure including energetic disorder. The mobility ratios quantify the effect of grain boundary on charge transport and are averages over 5 structures per angle. a) effect of the energy landscape, b) effect of disorder in electronic coupling and c) total mobility reduction factor due to grain boundary.

in Figure 3. Here, we consider 4 stripes for each of the 5 molecular assemblies per angle $\vartheta$ with ranges $x_{0}-x_{1}=$ $120-160 \mathrm{~nm}, 160-200 \mathrm{~nm}, 200-240 \mathrm{~nm}, 240-280 \mathrm{~nm}$, where $x$ is the axis parallel to the grain boundary. Since the origin of the energy barrier at the grain/grain interface is due to short-range interactions, we consider in the following only site energies from cutoff calculations. Compared to transport simulations on full, $400 \AA$ wide molecular structures, the variance of modelled mobilities is increased and calculated mobility ratios are larger (see Figure 3 and Appendix F).

The influence of the energy surface increases towards larger $\vartheta$, resulting in an average mobility ratio of over 20 for $\vartheta=90^{\circ}$. These simulations implicitly assume that the intergranular interface is too small for charge carriers to access a low-energy region of the boundary. As before, calculations including polarization result in higher mobilities compared to electrostatics-only simulations: $\mu_{X t a l} / \mu_{E=\text { const }}$ increases until 67.5 degrees and drops for 90 degrees. This behaviour might be explained in terms of high variances of modelled mobilities when considering $40 \AA$ wide stripes (see Appendix F). Therefore, we assume that the effect of electronic coupling might only increase slightly with $\vartheta$. Finally, the ratio between simulated mobilities with disorder in site energies and electronic couplings versus the mobility of ideal crystal structures increases with $\vartheta$ : For 90 degrees, the average mobility ratio $\mu_{X t a l} / \mu$ is more than 5 times higher than for 0 degrees. 
a)

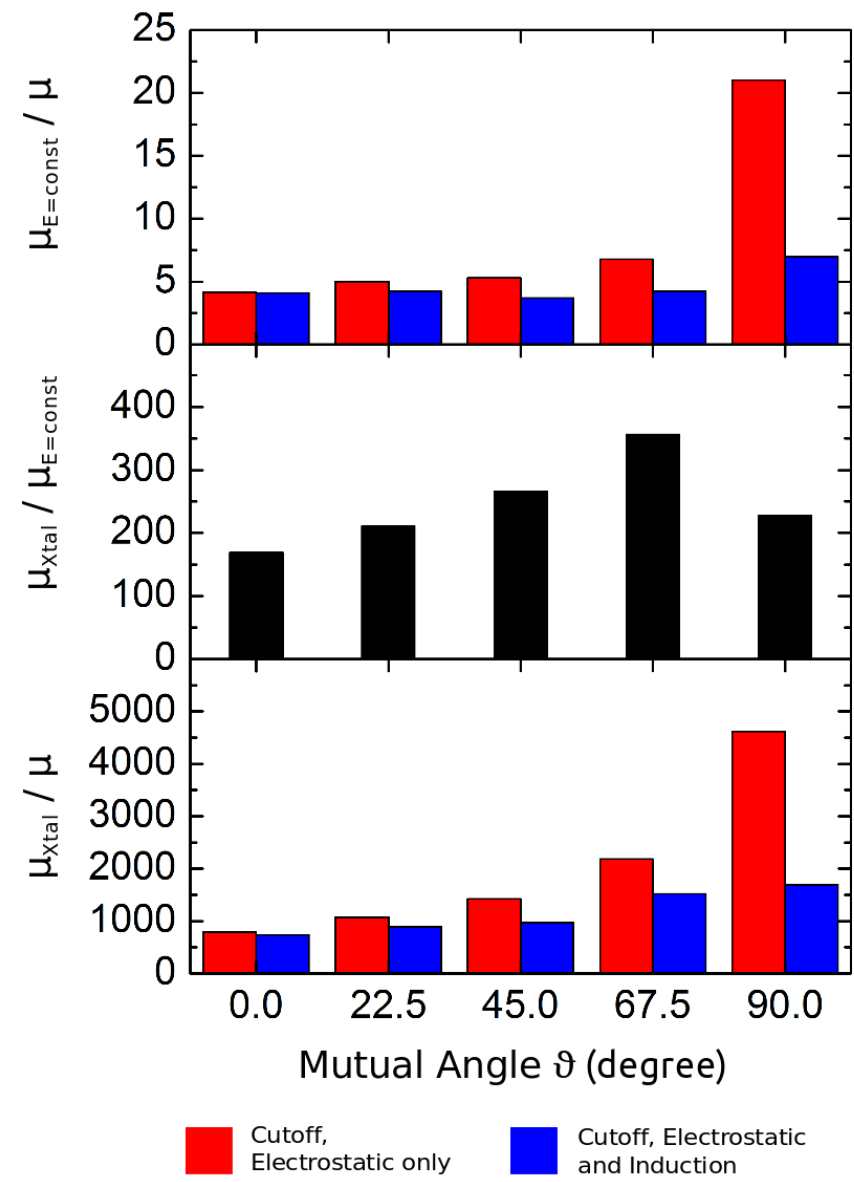

FIG. 3: Results of charge transport simulations of small, $4 \times 40 \mathrm{~nm}^{2}$ structures with one grain boundary and varied mutual crystal orientations. $\mu_{X t a l}$ refers to the mobility of the ideal crystal structure, $\mu_{E=\text { const }}$ relates to the mobility of the equilibrated molecular dynamics structure without energetic disorder and $\mu$ is the mobility of the equilibrated molecular dynamics structure including energetic disorder. The mobility ratios quantify the effect of grain boundary on charge transport and are averages over 20 structures per angle. a) effect of the energy landscape, b) effect of disorder in electronic coupling and c) total mobility reduction factor due to grain boundary. On the top and bottom graphs we obtain a clear trend towards large mutual angles.

\section{Energetics at Lattice Grain Boundaries in Small Molecular Systems}

In the atomistic model of transport across grain boundaries in TIPS-P, we found that electrostatic interactions invariably lead to an energy barrier at the grain/grain interface. Here, we will show that the creation of an energy barrier is, however, by no means universal: Notably, other interfacial energy profiles are conceivable, including trap and barrier/trap motifs.

As an abstraction of the complex atomistic description, we now employ a lattice model which (see also the Methods section) consists of probabilistically grown crystallites populated by quadrupolar particles with uniaxial symmetry. The molecular electrostatic layout is encoded in a single quadrupole component $Q_{20}=Q_{z z}<0$ associated, for example, with the normal of the conjugated plane in compounds such as pentacene or the long axis of small molecules with an acceptor-donor-acceptor architecture. We also note that such a representation is (almost) general as the quadrupole tensor in the eigenframe of a molecule has at best two non-zero components $\left(Q_{20}\right.$ and $\left.Q_{22 c}\right)$.

Snapshots of the lattice systems are shown in Fig. 4a-d, where the yellow stripes in the top panels indicate the orientation of the domains. The four panels refer to different seed numbers ( $n=2$ to 16 ) included here to rule out grain-size effects. The electrostatic contribution $\Delta_{h}^{(1)}$ to the site energy of holes is shown in the bottom panel, projected onto the $x y$-plane. First, it can be seen that the energy level within the domains is approximately constant. This intradomain energy is determined by long-range interactions primarily via the out-of-plane component of the quadrupole tensor, which is identical for all grains, as they share the same orientation with respect to the thin-film normal. The energetics at the grain/grain interface, on the other hand, is exclusively determined by the short-range interaction of the excess charge with the polar end groups of the neighbouring grain. Due to the crystal packing 

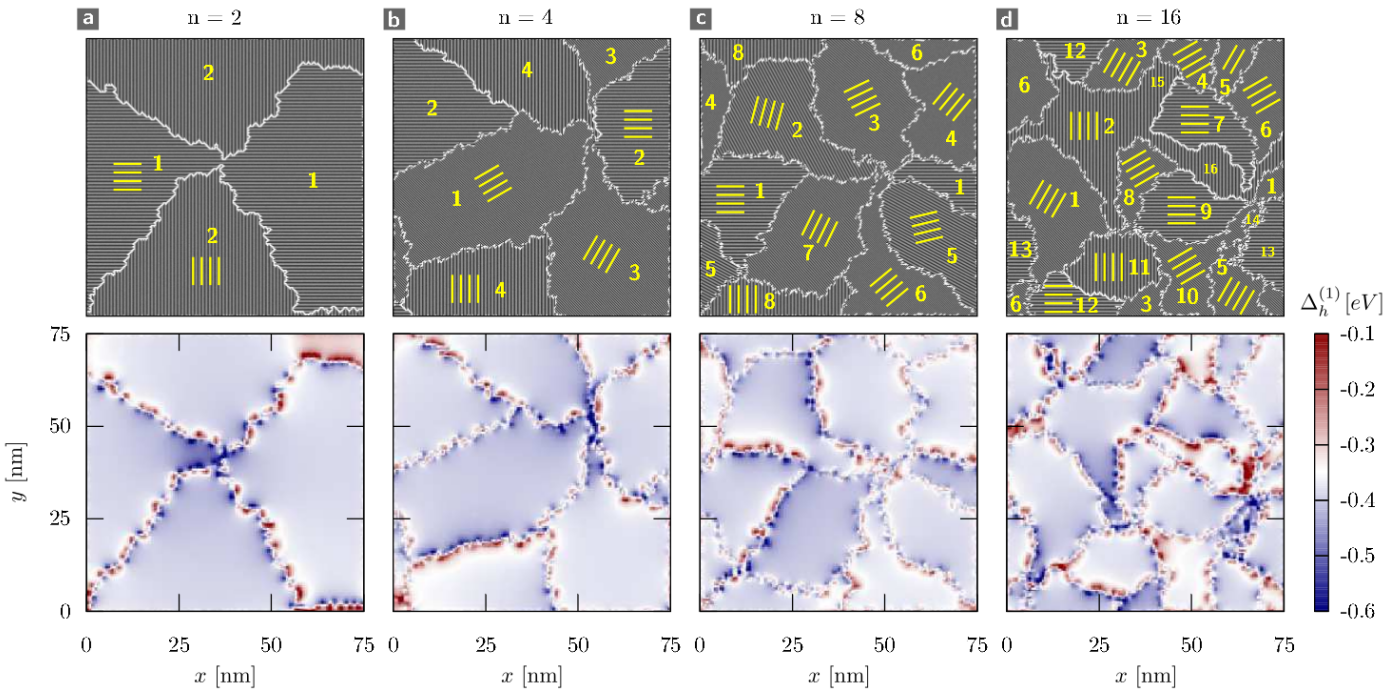

FIG. 4: Probabilistically grown $Q_{20} b c c$ lattice grain boundaries with varying seed density from $n=2$ (a) to $n=16$ (d) seeds. The line blocks in the top panel indicate the orientation of the grains such that the line direction coincides with the local axis of the quadrupolar particles associated with the $Q_{20}=-10$ au component. The bottom panel shows the electrostatic contribution to the energy landscape for holes projected onto the $x y$-plane.

and $Q_{20}<0$ character of the polar sites, the energy profile across the interface is characterized by the concurrent appearance of a barrier and an adjacent trap (referred to as the barrier/trap motif), with an only slight dependence of the barrier height and well depth on the grain/grain angle. This should be contrasted with the pure-barrier motif found for holes in TIPS-P (see above).

It is important to note that for electrons the energy landscape is reversed $\left(\Delta_{e}^{(1)}=-\Delta_{h}^{(1)}\right)$, as the formation of both barrier and barrier/trap structures is a result of the electrostatic (rather than polarization) contribution. This also implies that the high-energy states are not induced by a weaker dielectric stabilization due to interfacial voids. Changing from holes over to electrons, the barrier and barrier/trap therefore turn into a trap and trap/barrier motif, respectively. In the case of the lattice model from Fig. 4, charge-carrier migration across the boundary in the case of low carrier concentrations hence has to involve thermal activation independently of the type of the carrier. For TIPS$\mathrm{P}$, however, thermal activation is only required for electrons, which are likely to localize at an interfacial trap site. The grain/grain interface should therefore impact the effective activation energy for electron transport in TIPS-P, as probed by temperature- dependent mobility measurements. Holes, by contrast, can drift-diffuse through a gap in the barrier, since energetic disorder renders the boundary relatively porous, as demonstrated above for the case of large grain/grain interfaces.
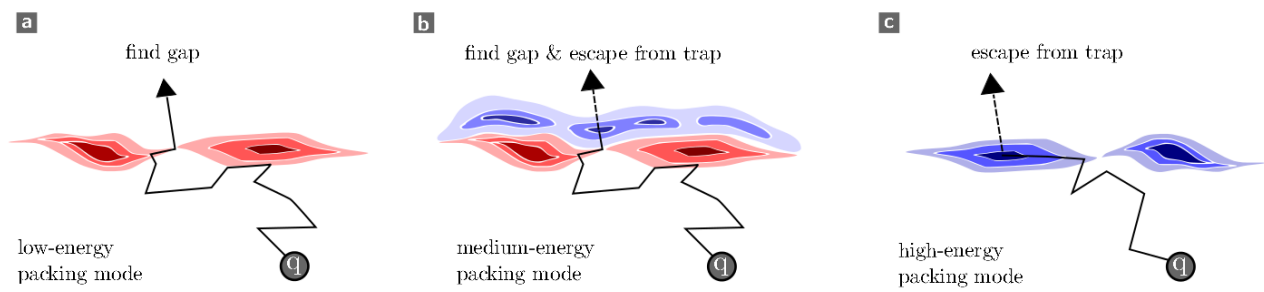

FIG. 5: Schematic of charge migration across a grain boundary for different interfacial energy profiles, including the (a) barrier, (b) barrier/trap and (c) trap motif.

Depending on molecular architecture and packing, all three interfacial energy motifs - barrier, trap, and barrier/trap (see Fig. 5) - are conceivable. For the barrier motif, migration across the grain boundary is limited by the time required to access a gap in the barrier, whereas for the well or barrier/well motif, the escape time from the trap serves as the controlling time scale for interdomain transport. The barrier motif is hence expected to exhibit the best transport properties. It is most likely to occur for low-energy packing modes (always defined with respect to one carrier type, electrons or holes), as the disruption of the molecular arrangement caused by the grain boundary then necessarily implies the formation of higher-energy states. Which packing mode (brickwork, herringbone, lamellar/columnar) 
presents a low-energy mode in turn depends on the molecular architecture. TIPS-P, for example, is characterized by a negative quadrupole component along the $\pi$-plane normal, next to a positive in-plane periphery composed of hydrogenated end groups. At the grain boundary, the bidirectional $\pi-\pi$ arrangement is lost, with the result that the positive end groups of the neighbouring grain destabilize hole carriers.

\section{Conclusion}

If grain/grain contact areas are large and without voids (with contact widths of $40 \mathrm{~nm}$ ), charge transport is unaffected by grain boundaries. Contrarily, as this contact area becomes smaller (widths of $4 \mathrm{~nm}$ ), mobility decreases severely towards larger grain/grain angles, which is mainly due to the formation of energy barriers. In general, stripes exhibit lower mobilities than full structures due to the confinement to higher-energy pathways for charge migration. Thus, in order to explain experimentally observed mobility reductions due to grain boundaries, grain/grain contact areas must be small. This might be the case if the boundaries contain voids or are built from small, interconnected grains as proposed by Wong et al. ${ }^{37}$ (see Appendix C). The formation of energy barriers at interfaces without voids is, however, by no means universal: The energy level profile across grain/grain interfaces can exhibit barrier, barrier/trap and trap motifs depending on molecular architecture, packing and charge-carrier type. For low chargecarrier concentrations, low-site-energy packing modes are most desirable, as the distortion of the supramolecular structure at the grain boundary then creates energy barriers made permeable by energetic disorder, thus providing a mobility-maintaining pathway for intergranular transport.

\section{Acknowledgements}

Florian Steiner would like to thank the Xerox Foundation / Xerox Research Centre of Canada (XRCC) and the DAAD for their financial support. Jenny Nelson thanks EPSRC (zone casting, EL, supergen project - numbers are missing) and the Royal Society Wolfson Research Merit Award. 
1 J. E. Anthony, J. S. Brooks, D. L. Eaton, and S. R. Parkin. Functionalized pentacene: improved electronic properties from control of solid-state order. J. Am. Chem. Soc., 123(38):9482-9483, Aug. 2001.

${ }^{2}$ G. Baccarani, B. Ricco, and G. Spadini. Transport properties of polycrystalline silicon films. Journal of applied physics, 49(11):5565-5570, 1978.

3 J.-L. Bredas, D. Beljonne, V. Coropceanu, and J. Cornil. Charge-transfer and energy-transfer processes in $\pi$-conjugated oligomers and polymers: a molecular picture. Chemical Reviews, 104(11):4971-5004, 2004. PMID: 15535639.

4 J.-F. Chang, T. Sakanoue, Y. Olivier, T. Uemura, M.-B. Dufourg-Madec, S. G. Yeates, J. Cornil, J. Takeya, A. Troisi, and H. Sirringhaus. Hall-effect measurements probing the degree of charge-carrier delocalization in solution-processed crystalline molecular semiconductors. Phys. Rev. Lett., 107(6):066601-, Aug. 2011.

${ }^{5}$ J. Chen, D. C. Martin, and J. Anthony. Morphology and molecular orientation of thin-film bis(tri-isopropylsilylethynyl) pentacene. Journal of Materials Research, 22:1701-1709, 2007.

6 J. Cornil, S. Verlaak, N. Martinelli, A. Mityashin, Y. Olivier, T. Van Regemorter, G. Davino, L. Muccioli, C. Zannoni, and F. Castet. Exploring the energy landscape of the charge transport levels in organic semiconductors at the molecular scale. Accounts of chemical research, 46(2):434-443, 2012.

7 G. D'Avino, S. Mothy, L. Muccioli, C. Zannoni, L. Wang, J. Cornil, D. Beljonne, and F. Castet. Energetics of electron-hole separation at p3ht/pcbm heterojunctions. The Journal of Physical Chemistry C, 117(25):12981-12990, 2013.

${ }^{8}$ R. De Boer, T. Klapwijk, and A. Morpurgo. Field-effect transistors on tetracene single crystals. Applied physics letters, 83(21):4345-4347, 2003.

${ }^{9}$ Y. Diao, B. C. Tee, G. Giri, J. Xu, D. H. Kim, H. A. Becerril, R. M. Stoltenberg, T. H. Lee, G. Xue, and S. C. Mannsfeld. Solution coating of large-area organic semiconductor thin films with aligned single-crystalline domains. Nature materials, 12(7):665-671, 2013.

10 A. Facchetti. Organic semiconductors: Made to order. Nature materials, 12(7):598-600, 2013.

11 A. A. Guilbert, J. M. Frost, T. Agostinelli, E. Pires, S. Lilliu, J. E. Macdonald, and J. Nelson. Influence of bridging atom and side chains on the structure and crystallinity of cyclopentadithiophenebenzothiadiazole polymers. Chemistry of Materials, 26(2):1226-1233, 2014.

12 R. L. Headrick, S. Wo, F. Sansoz, and J. E. Anthony. Anisotropic mobility in large grain size solution processed organic semiconductor thin films. Appl. Phys. Lett., 92(6):063302-3, Feb. 2008.

${ }^{13}$ G. Horowitz and M. Hajlaoui. Grain size dependent mobility in polycrystalline organic field-effect transistors. synthetic Metals, 122(1):185-189, 2001.

${ }^{14}$ G. Horowitz and M. E. Hajlaoui. Mobility in polycrystalline oligothiophene field-effect transistors dependent on grain size. Adv. Mater., 12(14):1046-1050, 2000.

15 W. L. Jorgensen, D. S. Maxwell, and J. Tirado-Rives. Development and testing of the opls all-atom force field on conformational energetics and properties of organic liquids. J. Am. Chem. Soc., 118(45):11225-11236, Jan. 1996.

${ }^{16}$ V. Kalihari, E. Tadmor, G. Haugstad, and C. D. Frisbie. Grain orientation mapping of polycrystalline organic semiconductor films by transverse shear microscopy. Advanced Materials, 20(21):4033-4039, 2008.

17 T. W. Kelley and C. D. Frisbie. Gate voltage dependent resistance of a single organic semiconductor grain boundary. The Journal of Physical Chemistry B, 105(20):4538-4540, 2001.

18 J. Kirkpatrick. An approximate method for calculating transfer integrals based on the zindo hamiltonian. Int. J. Quantum Chem., 108(1):51-56, 2008.

19 J. J. Kwiatkowski, J. M. Frost, and J. Nelson. The effect of morphology on electron field-effect mobility in disordered c60 thin films. Nano Letters, 9(3):1085-1090, 2009. PMID: 19275245.

20 J. Lee, S. Roth, and Y. Park. Anisotropic field effect mobility in single crystal pentacene. Applied physics letters, 88(25):252106-252106-3, 2006.

${ }^{21}$ S. S. Lee, J. M. Mativetsky, M. A. Loth, J. E. Anthony, and Y.-L. Loo. Quantifying resistances across nanoscale low-and high-angle interspherulite boundaries in solution-processed organic semiconductor thin films. ACS nano, 6(11):9879-9886, 2012.

22 J. Levinson, F. R. Shepherd, P. J. Scanlon, W. D. Westwood, G. Este, and M. Rider. Conductivity behavior in polycrystalline semiconductor thin film transistors. Journal of Applied Physics, 53(2):1193-1202, 1982.

${ }^{23} \mathrm{H}$. Mathieu and H. Fanet. Physique des semiconducteurs et des composants electroniques: Cours et exercices corriges. DUNOD, 6eme edition, 2009.

24 J. Nelson, J. J. Kwiatkowski, J. Kirkpatrick, and J. M. Frost. Modeling charge transport in organic photovoltaic materials. Accounts of Chemical Research, 42(11):1768-1778, 2009. PMID: 19848409.

${ }^{25}$ O. Ostroverkhova, D. G. Cooke, F. A. Hegmann, R. R. Tykwinski, S. R. Parkin, and J. E. Anthony. Anisotropy of transient photoconductivity in functionalized pentacene single crystals. Applied Physics Letters, 89(19):192113, 2006.

26 J. Pereira, C. Catlow, and G. Price. Molecular dynamics simulation of methanolic and ethanolic silica-based sol-gel solutions at ambient temperature and pressure. The Journal of Physical Chemistry A, 106(1):130-148, 2002.

27 C. Poelking, M. Tietze, C. Elschner, S. Olthof, D. Hertel, B. Baumeier, F. Wrthner, K. Meerholz, K. Leo, and D. Andrienko. Impact of mesoscale order on open-circuit voltage in organic solar cells. Nature materials, 2014.

${ }^{28}$ K. Puntambekar, J. Dong, G. Haugstad, and C. D. Frisbie. Structural and electrostatic complexity at a pentacene/insulator interface. Adv. Funct. Mater., 16(7):879-884, 2006.

29 J. Rivnay, L. H. Jimison, J. E. Northrup, M. F. Toney, R. Noriega, S. Lu, T. J. Marks, A. Facchetti, and A. Salleo. Large 
modulation of carrier transport by grain-boundary molecular packing and microstructure in organic thin films. Nat Mater, 8(12):952-958, Dec. 2009.

30 J. Rivnay, S. C. B. Mannsfeld, C. E. Miller, A. Salleo, and M. F. Toney. Quantitative determination of organic semiconductor microstructure from the molecular to device scale. Chem. Rev., pages -, Aug. 2012.

31 V. Ruehle, C. Junghans, A. Lukyanov, K. Kremer, and D. Andrienko. Versatile object-oriented toolkit for coarse-graining applications. J. Chem. Theory Comput., 5(12):3211-3223, Nov. 2009.

32 T. Sakanoue and H. Sirringhaus. Band-like temperature dependence of mobility in asolution-processed organic semiconductor. Nat Mater, 9(9):736-740, Sept. 2010.

33 J. Y. Seto. The electrical properties of polycrystalline silicon films. Journal of Applied Physics, 46(12):5247-5254, 1975.

34 J. Soeda, T. Uemura, T. Okamoto, C. Mitsui, M. Yamagishi, and J. Takeya. Inch-size solution-processed single-crystalline films of high-mobility organic semiconductors. Applied Physics Express, 6(7):076503, 2013.

${ }^{35}$ F. Steiner, S. Foster, A. Losquin, J. Labram, T. D. Anthopoulos, J. M. Frost, and J. Nelson. Distinguishing the influence of structural and energetic disorder on electron transport in fullerene multi-adducts. Materials Horizons, 2(1):113-119, 2015.

36 J. Wade, F. Steiner, D. Niedzialek, D. T. James, Y. Jung, D.-J. Yun, D. D. Bradley, J. Nelson, and J.-S. Kim. Charge mobility anisotropy of functionalized pentacenes in organic field effect transistors fabricated by solution processing. Journal of Materials Chemistry C, 2(47):10110-10115, 2014.

37 C. Y. Wong, B. L. Cotts, H. Wu, and N. S. Ginsberg. Exciton dynamics reveal aggregates with intermolecular order at hidden interfaces in solution-cast organic semiconducting films. Nature communications, 6, 2015. 


\section{Supporting Information}

\section{Appendix A: Force-Field of TIPS-P}

For atomistic molecular-dynamics simulations a force-field specific to TIPS-P is developed. The OPLS-AA forcefield

$$
\begin{aligned}
E= & \sum_{i<j} 4 \epsilon_{i j}\left[\left(\frac{\sigma_{i j}}{r_{i j}}\right)^{12}-\left(\frac{\sigma_{i j}}{r_{i j}}\right)^{6}\right]+\sum_{i<j} \frac{1}{4 \pi \epsilon_{r} \epsilon_{0}} \frac{q_{i} q_{j}}{r_{i j}} \\
& \sum_{b} \frac{1}{2} K_{b}\left(b-b_{0}\right)^{2}+\sum_{\theta} \frac{1}{2} K_{\theta}\left(\theta-\theta_{0}\right)^{2} \\
& \sum_{\xi} \frac{1}{2} K_{\xi}\left(\xi-\xi_{0}\right)^{2}+\sum_{n} c_{n} \cos ^{n}\left(\phi-\phi_{0}\right)
\end{aligned}
$$

was adopted where the first two terms represent non-bonding interactions (Lennard-Jones and Coulomb potentials) and the last four terms relate to bonding interactions (bond, angle, improper dihedrals and dihedrals) ${ }^{15}$. For TIPS-P we define seven different atom identities that refer to OPLS-AA atom types (see Table I).

$\begin{array}{llll}\text { atom identity opls number } & \text { atom type } & \text { in forcefield } \\ \text { Cxx } & \text { opls_135 } & \text { alkane CH3 CT } \\ \text { Cx } & \text { opls_136 } & \text { alkane CH2 } & \text { CT } \\ \text { Hx or Hxx } & \text { opls_140 } & \text { alkane H } & \text { HC } \\ \text { CAx } & \text { opls_145 } & \text { benzene C } & \text { CA } \\ \text { HAx } & \text { opls_146 } & \text { benzene H } & \text { HA } \\ \text { CTx } & \text { opls_925 } & \text { alkyne } & \text { CZ } \\ \text { Six } & \text { opls_966 } & \text { silicon } & \text { SI }\end{array}$

TABLE I: Symbols used in Figure 6 according OPLS-AA number and atom type. $x$ stands for an arbitrary integer.

A Mulliken population analysis was conducted to compute atomic charges. The molecular geometry was optimised and atomic charges were calculated with hybrid density functional theory employing the hybrid functional B3LYP and a $6-311 \mathrm{~g}^{* *}$ basis set. The results are given in Table II. For all carbon and hydrogen atoms masses and Lennard-Jones parameters are taken from the OPLS-AA force-field. For silicon we use the Lennard-Jones parameters provided in reference $^{26}$ (SI: $\epsilon_{i i}=0.398 \mathrm{~kJ} / \mathrm{mol}$ and $\sigma_{i i}=0.4435 \mathrm{~nm}$ ).

In the following we discuss specifications for bond types, angle types and dihedral types (see Tables III, IV and V. Parameters for bonds, angles and dihedrals between carbon and hydrogen atoms can be found in the OPLS-AA database and are not discussed here. Parameters concerning silicon are provided in the tables mentioned above.

Since silicon is a four-bonding atom like carbon, we consider for angles and dihedrals containing silicon OPLS-AA parameters for alkanic carbon $(\mathrm{CT})$ or rely on previous parameters provided by Guilbert and co-workers ${ }^{11}$ (see Tables IV and V). In terms of bonds, reference ${ }^{11}$ provides the equilibrium bond length $b_{0}$ and the force constant $K_{b}$ for SI-CT. In contrast, the specifications of the bond SI-CZ are unknown.

We geometrically optimise TIPS-P with hybrid density functional theory (B3LYP/6-311g**). Accordingly, we iteratively vary the equilibrium bond length and the force constant of the SI-CZ bond in the force-field until all bond lengths agree in the molecular geometries from density functional theory and molecular mechanics. The result is provided in Table III. Thereafter, the force-field was tested on a $3 \times 3$ TIPS-P supercell. We found that the supercell and the molecular geometries were stable. 


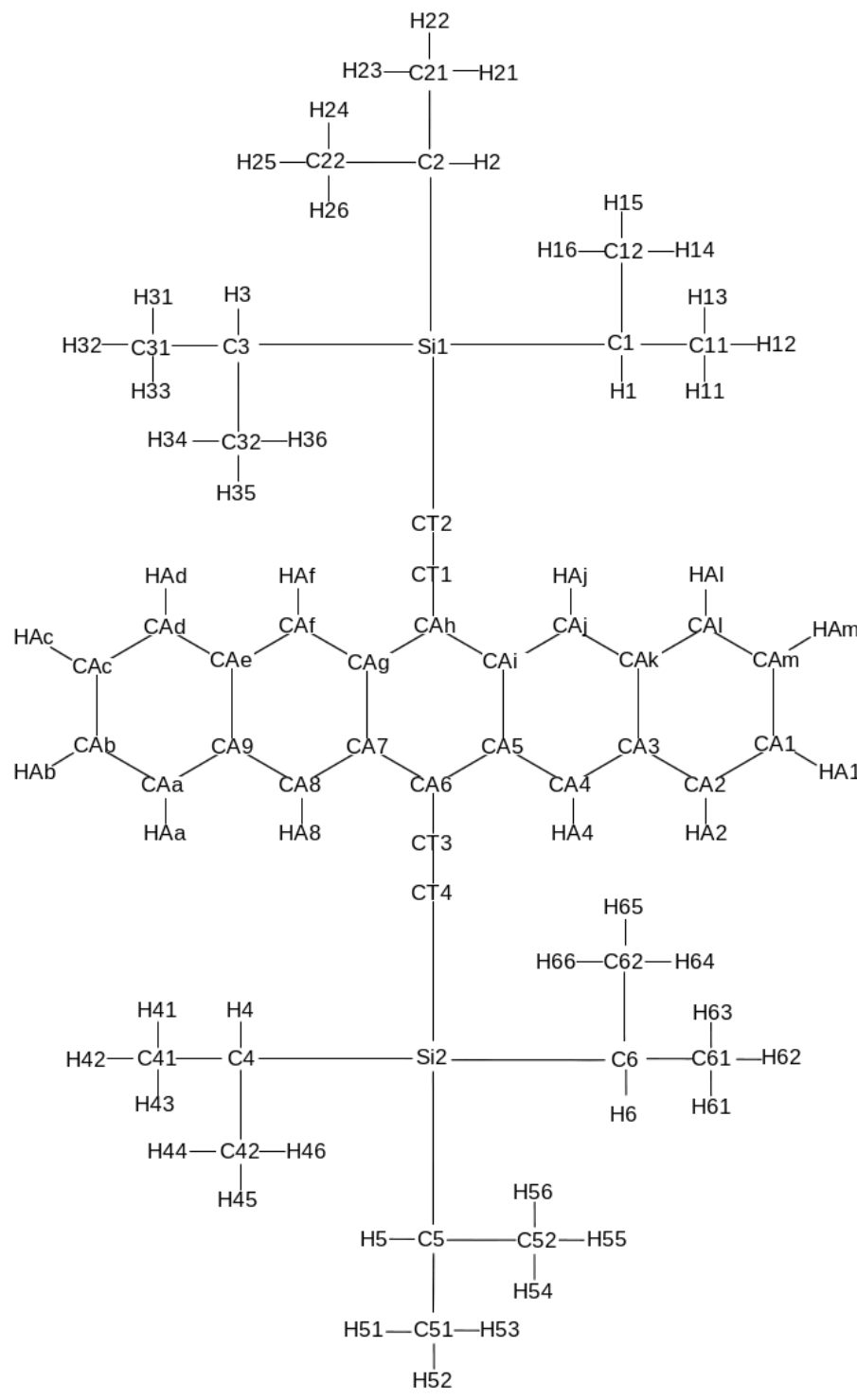

FIG. 6: Molecular structure of TIPS-P with symbols for each atom used for molecular dynamics simulations.

\section{Appendix B: Projective Method versus Molecular Oribital Overlap}

In this study, transfer integrals are calculated with the Molecular Orbital Overlap (MOO) method ${ }^{18}$. As discussed by Kirkpatrick, MOO is considerably faster than the projective method since self-consistent density functional theory calculation on the molecular pair can be omitted. MOO is based on the semi-empirical functional ZINDO (Zerner's Intermediate Neglect of Differential Overlap) which can not treat heavy atoms such as silicon.

Replacing silicon with hydrogen and neglecting the rest of the side chains allows the computation of transfer integrals with MOO (see Figure 7b). We belief that this is an appropriate approximation since charge transport occurs between the $\pi$-systems of TIPS-P which is the pentacene unit. This agrees with the location of the HOMO level (see Figure 7c).

Table VI compares transfer integrals calculated with the projective method on the full molecular structure of TIPS-P (Figure 7a) and with MOO on the reduced structure (Figure 7b). Transfer integrals computed with MOO are slightly higher than for the projective method. The two-dimensional nature of charge transport in TIPS-P is preserved when calculating transfer integrals with MOO. 


\begin{tabular}{|c|c|c|c|}
\hline atom identity & Mulliken charge (C) & atom identity & Mulliken charge \\
\hline CA 6 & -0.037903 & $\mathrm{C} 61$ & -0.280104 \\
\hline CA5 & -0.024736 & $\mathrm{H} 61$ & 0.107321 \\
\hline $\mathrm{CAi}$ & -0.017368 & $\mathrm{H} 62$ & 0.104415 \\
\hline $\mathrm{CAh}$ & -0.031665 & $\mathrm{H} 63$ & 0.104267 \\
\hline CA4 & 0.015652 & $\mathrm{C} 62$ & -0.281669 \\
\hline $\mathrm{CAj}$ & 0.014919 & $\mathrm{H} 64$ & 0.101288 \\
\hline $\mathrm{CAk}$ & -0.050873 & H65 & 0.100655 \\
\hline $\mathrm{CA} 3$ & -0.062843 & $\mathrm{H} 66$ & 0.118958 \\
\hline $\mathrm{CA} 2$ & -0.068552 & $\mathrm{C} 41$ & -0.281219 \\
\hline HA2 & 0.090358 & $\mathrm{H} 41$ & 0.105783 \\
\hline CA1 & -0.091045 & $\mathrm{H} 42$ & 0.104058 \\
\hline $\mathrm{CAm}$ & -0.088084 & $\mathrm{H} 43$ & 0.107806 \\
\hline $\mathrm{CAl}$ & -0.061955 & $\mathrm{C} 42$ & -0.280412 \\
\hline HA4 & 0.133049 & $\mathrm{H} 44$ & 0.117911 \\
\hline $\mathrm{HAj}$ & 0.117454 & H45 & 0.100254 \\
\hline HA1 & 0.095289 & $\mathrm{H} 46$ & 0.106212 \\
\hline $\mathrm{HAm}$ & 0.095368 & C51 & -0.287312 \\
\hline $\mathrm{HAl}$ & 0.084265 & H51 & 0.110784 \\
\hline CA7 & -0.011951 & $\mathrm{H} 52$ & 0.102495 \\
\hline $\mathrm{CA} 8$ & 0.015823 & H53 & 0.110066 \\
\hline $\mathrm{CAg}$ & -0.021739 & $\mathrm{C} 52$ & -0.285191 \\
\hline HA 8 & 0.123179 & $\mathrm{H} 54$ & 0.116829 \\
\hline $\mathrm{CAf}$ & 0.010026 & H55 & 0.102296 \\
\hline HAf & 0.137156 & H56 & 0.105284 \\
\hline CA9 & -0.053162 & $\mathrm{C} 3$ & -0.50675 \\
\hline $\mathrm{CAa}$ & -0.063648 & $\mathrm{H} 3$ & 0.14054 \\
\hline $\mathrm{CAe}$ & -0.063644 & C31 & -0.276603 \\
\hline $\mathrm{CAb}$ & -0.0888 & H31 & 0.104923 \\
\hline $\mathrm{HAa}$ & 0.086942 & H32 & 0.106576 \\
\hline $\mathrm{CAd}$ & -0.068506 & H33 & 0.105829 \\
\hline $\mathrm{CAc}$ & -0.090646 & $\mathrm{C} 32$ & -0.291701 \\
\hline $\mathrm{HAb}$ & 0.095537 & $\mathrm{H} 34$ & 0.104434 \\
\hline HAd & 0.08922 & H35 & 0.10226 \\
\hline $\mathrm{HAc}$ & 0.09533 & H36 & 0.118824 \\
\hline CT1 & 0.018347 & $\mathrm{C} 21$ & -0.279957 \\
\hline $\mathrm{CT} 2$ & -0.533583 & $\mathrm{H} 21$ & 0.099471 \\
\hline Si1 & 1.248732 & $\mathrm{H} 22$ & 0.099909 \\
\hline C1 & -0.501642 & $\mathrm{H} 23$ & 0.123337 \\
\hline $\mathrm{H} 1$ & 0.133081 & $\mathrm{C} 22$ & -0.279816 \\
\hline $\mathrm{C} 2$ & -0.511432 & $\mathrm{H} 24$ & 0.106234 \\
\hline $\mathrm{H} 2$ & 0.135603 & $\mathrm{H} 25$ & 0.105338 \\
\hline CT3 & -0.01656 & $\mathrm{H} 26$ & 0.106848 \\
\hline CT4 & -0.479566 & C11 & -0.281127 \\
\hline $\mathrm{Si} 2$ & 1.227914 & H11 & 0.104692 \\
\hline $\mathrm{C} 4$ & -0.501848 & $\mathrm{H} 12$ & 0.110457 \\
\hline $\mathrm{H} 4$ & 0.128708 & H13 & 0.103015 \\
\hline C5 & -0.497896 & $\mathrm{C} 12$ & -0.289291 \\
\hline H5 & 0.128431 & $\mathrm{H} 14$ & 0.102442 \\
\hline C6 & -0.510106 & H15 & 0.103687 \\
\hline $\mathrm{H} 6$ & 0.137179 & H16 & 0.117847 \\
\hline
\end{tabular}

TABLE II: Mulliken charges per atom.

$\begin{array}{lccl}\text { bond } & \mathrm{b}_{0}(\mathrm{~nm}) & \mathrm{K}_{b}\left(\mathrm{~kJ} / \mathrm{mol} / \mathrm{nm}^{2}\right) & \text { source } \\ \text { SI-CT } & 0.186 & 132334 & \text { from }^{11} \\ \text { SI-CZ } & 0.186 & 900000 & \text { fitted iteratively }\end{array}$

TABLE III: Bond-specific parameters.

$\begin{array}{lccl}\text { angle } & \theta_{0}\left({ }^{\circ}\right) & \mathrm{K}_{\theta}\left(\mathrm{kJ} / \mathrm{mol} / \mathrm{rad}^{2}\right) & \text { source } \\ \text { SI-CT-CT } & 116.000 & 488.273 & \text { OPLS from CT CT CT } \\ \text { SI-CT-HC } & 103.500 & 313.800 & \text { OPLS from CT CT HC } \\ \text { CT-SI-CT } 134.059 & 261.217 & \text { from }{ }^{11} \\ \text { SI-CZ-CZ } & 179.000 & 1255.20 & \text { OPLS from CT CZ CZ } \\ \text { CT-SI-CZ } & 105.000 & 488.273 & \text { OPLS from CT CT CZ }\end{array}$

TABLE IV: Angle-specific parameters.

\section{Appendix C: Grain Boundaries in TIPS-P as a Network of Small Grains}

Wong et al. suggested in reference ${ }^{37}$ that grain boundaries in TIPS-P are built from small crystals that are connected to each other by small interfaces. In this section, we study the influence of such a configuration on charge transport. As the interconnectivity between small grains in the grain boundary is unknown, we consider 4 different cases: i) a one-dimensional chain with two connections per grain, ii) a two-dimensional honeycomb structure with three connections per grain, iii) a two-dimensional quadratic lattice with four connections per grain and iv) a two-dimensional hexagonal structure with six connections per grain (see Figure 8a). The distance between next neighbors is set to 1 and model structures are $20 \times 20$ large.

Each grain is approximated as a hopping-site during charge transport. This assumes that charge transport is limited by grain boundaries and not by the mobility anisotropy within TIPS-P grains (see reference ${ }^{36}$ ). In our model we consider small interfaces between grains. This allows us to use the results of the previous section for $40 \AA$ wide 


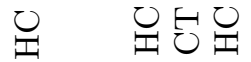

벙 烏兒兒

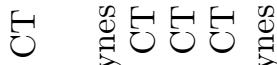

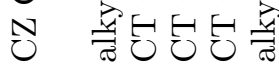

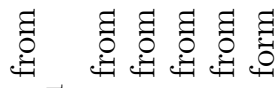

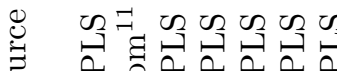

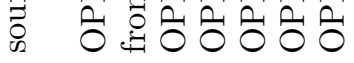

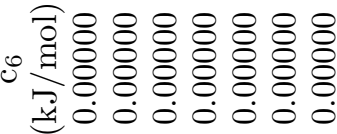

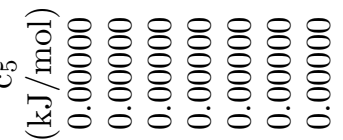

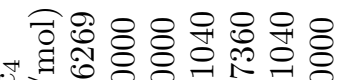

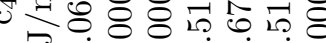

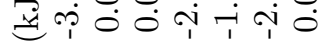

ও

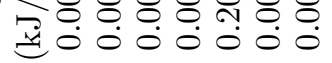

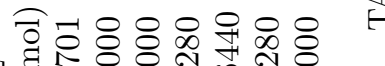

ن

島

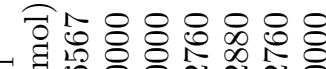

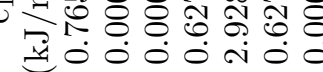

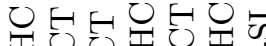

1

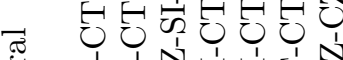

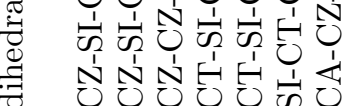


a)

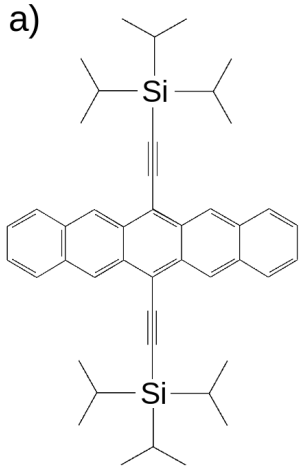

b)

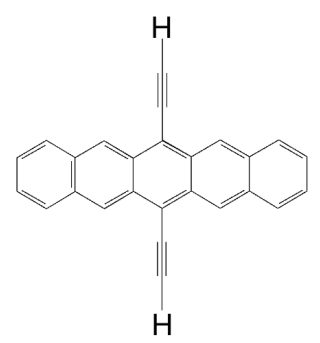

c)

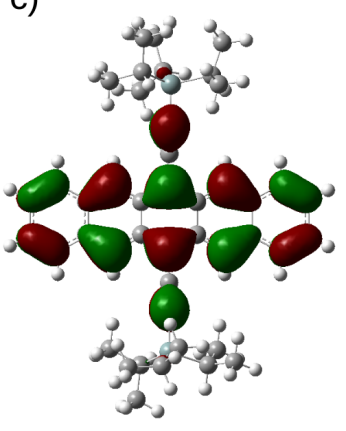

FIG. 7: a) Full molecular structure of TIPS-P and b) simplified structure (right) for transfer integral calculations with MOO. c) HOMO of TIPS-P calculated with the hybrid functional B3LYP and the basis set 6-311g**.

$\begin{array}{ccc}\text { vector } & \begin{array}{c}\mathrm{J}_{\text {ProjectiveMethode }} \\ (\mathrm{meV})\end{array} & \begin{array}{c}\mathrm{J}_{M O O} \\ (\mathrm{meV})\end{array} \\ \mathbf{a} & 53.7 & 85.7 \\ \mathbf{b} & 0.21 & 0.71 \\ \mathbf{c} & 1.17 & 2.13 \\ \mathbf{a - b} & 64.8 & 70.0\end{array}$

TABLE VI: Transfer integrals calculated with the molecular orbital overlap method (MOO) and the projective method ${ }^{18}$. $\mathbf{a}$, $\mathbf{b}$ and $\mathbf{c}$ are the unit cell vector of TIPS-P from reference ${ }^{1}$

stripes. For each angle $\vartheta$ we computed 20 reduction factors $\mu_{X t a l} / \mu$ describing the mobility reduction imposed by the grain boundary. Those are randomly assigned to each grain boundary between nano-crystals reducing the hopping rate from one grain to another by the drawn factor $\mu_{X t a l} / \mu$.

In terms of charge transport we consider two cases: a) all grains are well connected as if there are no grain boundaries in between them and b) the reduction factor $\mu_{X \text { tal }} / \mu$ is randomly drawn from all mutual angles $\left[0^{\circ}, 90^{\circ}\right]$; electrostatic only and electrostatic and induction are considered. All reduction factors are corrected by the mobility ratio $\mu_{X \text { tal }} / \mu$ of the complete, $400 \times 400 \AA^{2}$ structures with parallel grains since this is the case where both grains crystalized, charge transport is unconfined and only disorder within the grain plays a role.

a)

2
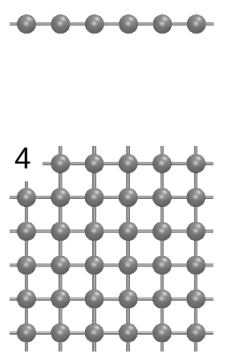

3
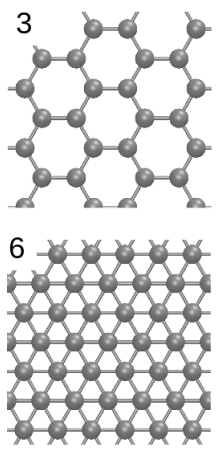

b)

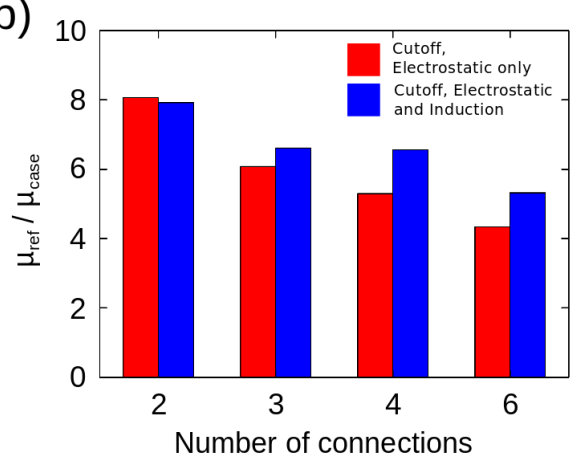

FIG. 8: Grain boundaries as a network of nano-crystals. a) Model for nano-crystals with different connectivity to neighbouring grains. Spheres represent grains and bonds refer to connections between spheres. The number indicates how many next neighbours there are. b) Mobility ratios between a perfectly connected grid $\mu_{r e f}$ and a network with variation in connectivity $\mu_{\text {case }}$. Reduced connection due to grain boundaries are represented by individual (not the average) mobility reductions factors from $4 \times 40 \mathrm{~nm}^{2}$ stipe structures. All angles are considered.

The results of kinetic Monte-Carlo simulations are provided in Figure 8b. Mobilities are calculated from average 
velocities utilising regenerative contacts. The reference mobility $\mu_{r e f}$ refers to the case of perfectly connected grains while the mobility $\mu_{\text {case }}$ relates to the case of reduced connectivity between grains due to small, $4 \mathrm{~nm}$ wide grain boundaries (see cases a) and b) from above). The higher the number of connections between grains the lower is the effect of grain boundaries reaching from a mobility ratio $\mu_{\text {ref }} / \mu_{\text {case }}$ of around 8 to 4 for two and six connections respectively. More connections allow charge carriers to avoid grain boundaries with a strong blocking effect.

Appendix D: Energy Surface for Varied Mutual Angles
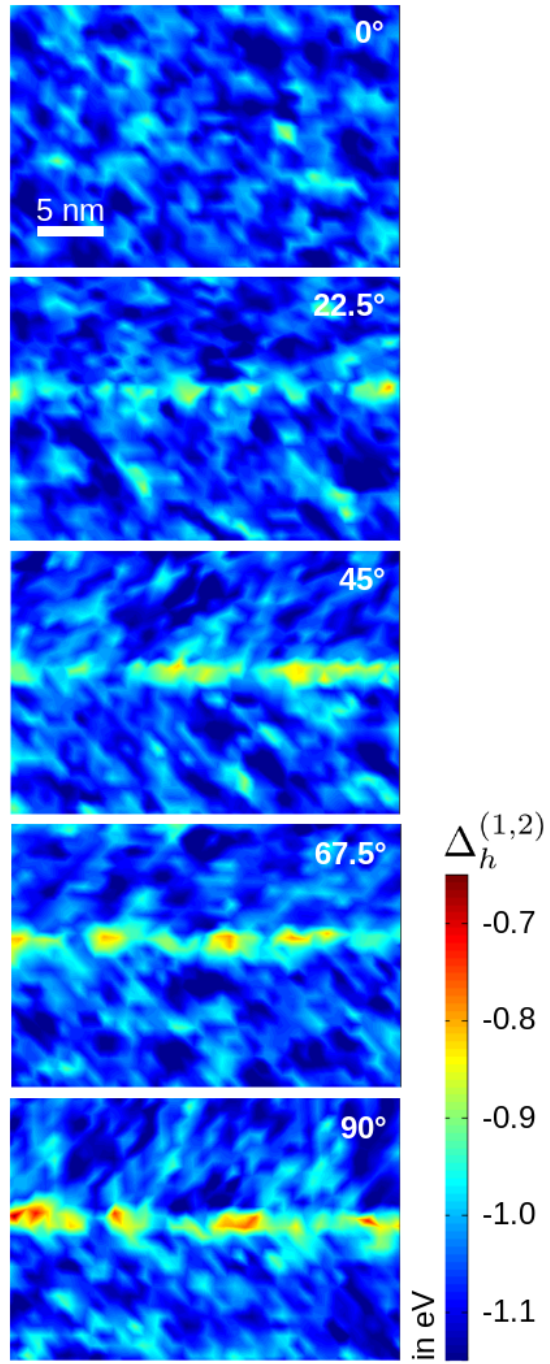

FIG. 9: Energy surfaces of representative two-grain structures for each mutual angle. Energy calculations consider contributions from electrostatics and induction.

\section{Appendix E: Transfer Integral Distribution for Varied Mutual Angles}

\section{Appendix F: Statistics for Two Grains with Varied Mutual Angle}

In the main text, charge transport is simulated for large, quadratic structures $\left(40 \mathrm{x} 40 \mathrm{~nm}^{2}\right)$ and stripes perpendicular to the grain boundary $\left(4 \times 40 \mathrm{~nm}^{2}\right)$. For large structures, we considered 5 molecular assemblies per angle while we simulated charge transport on 20 stripes per angle. Mobilities resulting from kinetic Monte-Carlo vary. This 


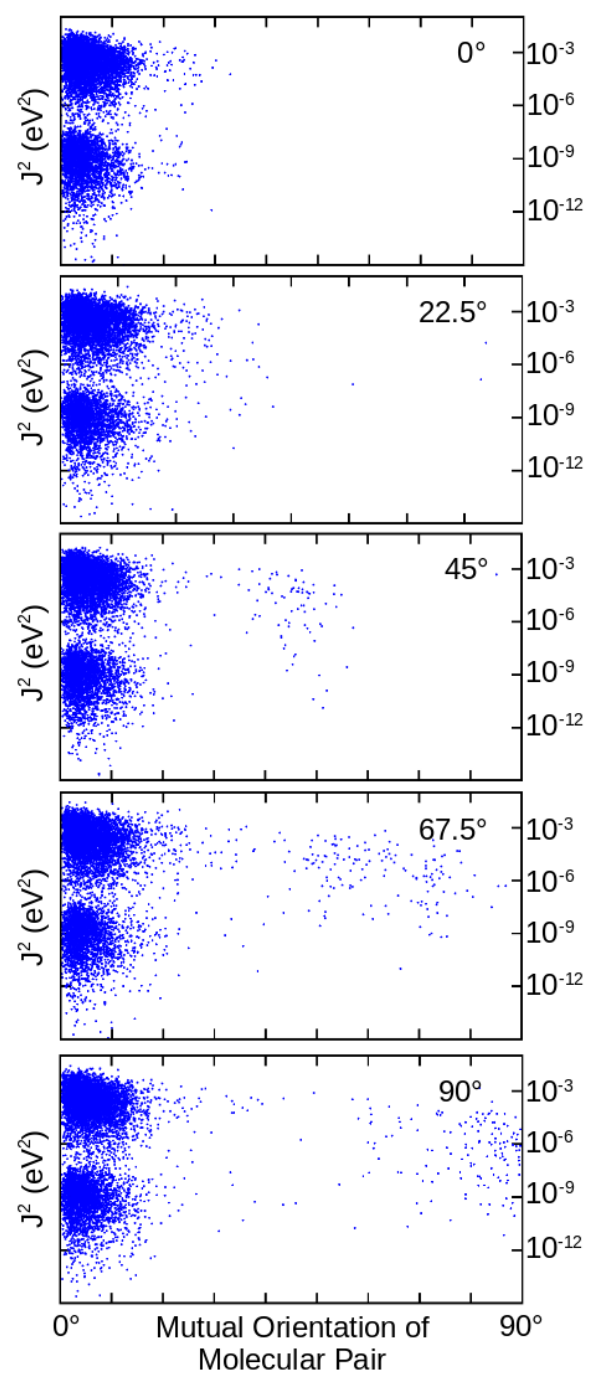

FIG. 10: Transfer integrals calculated for representative two-grain structures for each mutual angle.

section aims to quantify this variation. The used metric are mobility ratios of the ideal crystal $\mu_{X \text { tal }}$, the molecular dynamics molecular assemblies without energetic disorder $\mu_{E=\text { const }}$ or the molecular dynamics molecular structures with energetic disorder $\mu$ (with different cases for site energies).

For the large, $40 \times 40 \mathrm{~nm}^{2}$ structures the standard deviations of the mobility ratios are relatively small compared to the average value (see Figure 11). In contrast, the values of the standard deviations of the mobility ratios for stripes are similar to their average values (see Figure 12 ). In the case of $\mu / \mu_{E=\text { const }}$ standard deviations increase towards large mutual angles. This can be explained by the non-uniform barrier height between grains combined with the increase peak barrier heights for larger mutual angles.

Thus, the average mobility ratio values for large structures are very reliable. Large interfaces between TIPS-P grains do not seem to impede charge transport. For small inter-granular interfaces charge carrier mobilities are on average more reduced than for large mutual angles. Due to the large standard deviation, charge transport across small grain boundaries might be much better or worse. A simple model, how this variance will affect charge transport in nano-crystalline grain interfaces in TIPS-P is provided in Appendix C. 


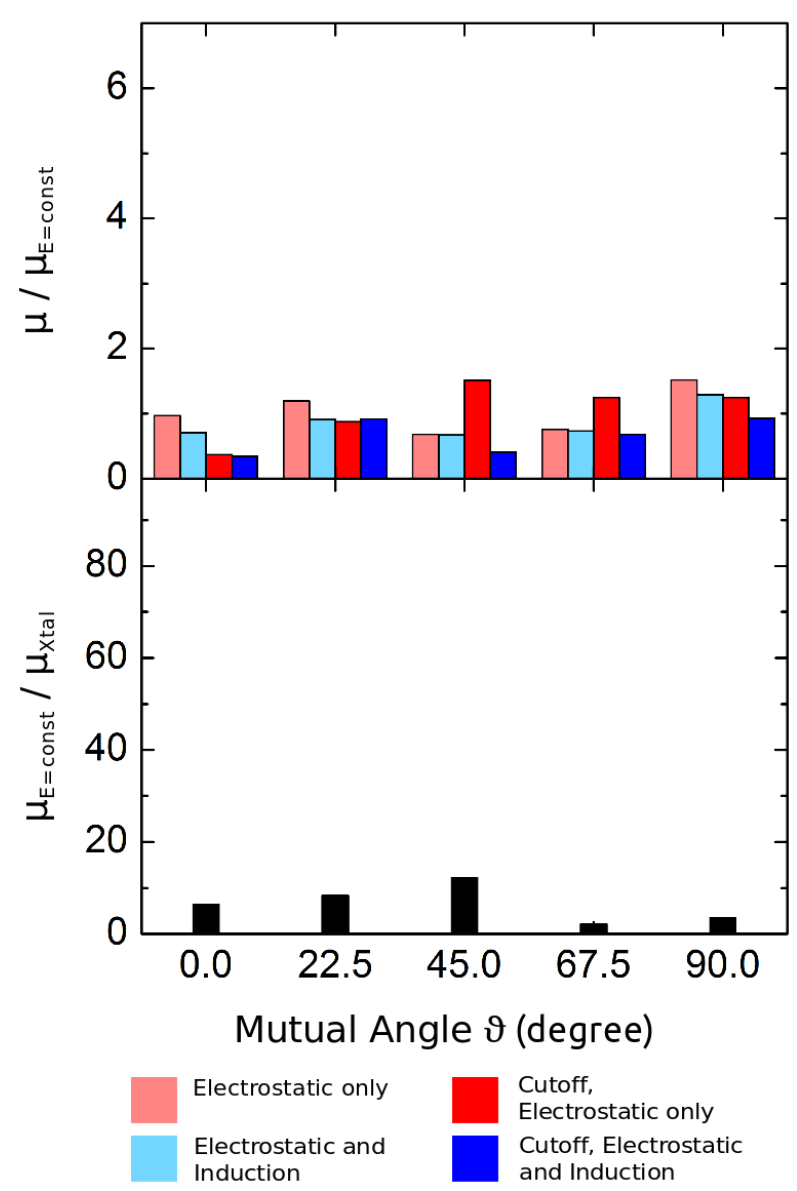

FIG. 11: Standard deviations of mobility ratios for large, $40 \times 40 \mathrm{~nm}^{2}$ structures. $\mu_{X t a l}$ refers to the mobility of ideal crystal structure $^{1}, \mu_{E=\text { const }}$ relates to the mobility of the molecular dynamics molecular assembly without energetic disorder and $\mu$ is the mobilty of the molecular dynamics molecular assembly including energetic disorder considering different cases.

\section{Appendix G: Randomly Grown Multi-Grain Systems of TIPS-P}

In the main text, structures with two grains and one linear grain boundary are simulated. Here, we model probabilistically grown, multi-grain structures. Charge transport parameters and charge transport is simulated as described in the main text. Furthermore, we track charge carriers and compute their accumulated occupation time per site allowing for a characterisation of the general charge transport behaviour in multi-crystalline films of TIPS-P.

Molecular assemblies are initially prepared with an algorithm probabilistically simulating the growth of grains. At first, the number of grains is chosen. Accordingly, seeds are randomly placed on a two-dimensional surface. Seeds are molecules that are oriented with $0,22.5,45,67.5$ or 90 degrees compared to the a-vector of the TIPS-P unit cell. Grains can grow in the $\mathbf{a}$ and $\mathbf{b}$ direction of the TIPS-P crystal structure. The initial seeds are the first generation of molecules. For every following generation, the probability to grow is reduced by a factor of 10 ensuring grains of similar size. The code avoids overlapping of molecules when one grain reaches another one. The process of placing molecules is repeated until a predefined number of molecules has been placed. Accordingly, the molecular assemblies are equilibrated with atomistic molecular dynamics as described in the main text for two-grain systems. An example structure is provided in Figure 13a.

The energy surface referring to calculations including electrostatics and induction is depicted in Figure 13b. Energy barriers between grains are particularly high for interfaces with large mutual angle. Moreover, we observe high potential energies for hole transport when three grains join each other. This is not surprising as the multipole moments of the three grains are not aligned. Additionally, voids distort the energy surface and significantly influencing the site energies of adjacent molecules. As mentioned previously, the energy barriers are non-uniform and 


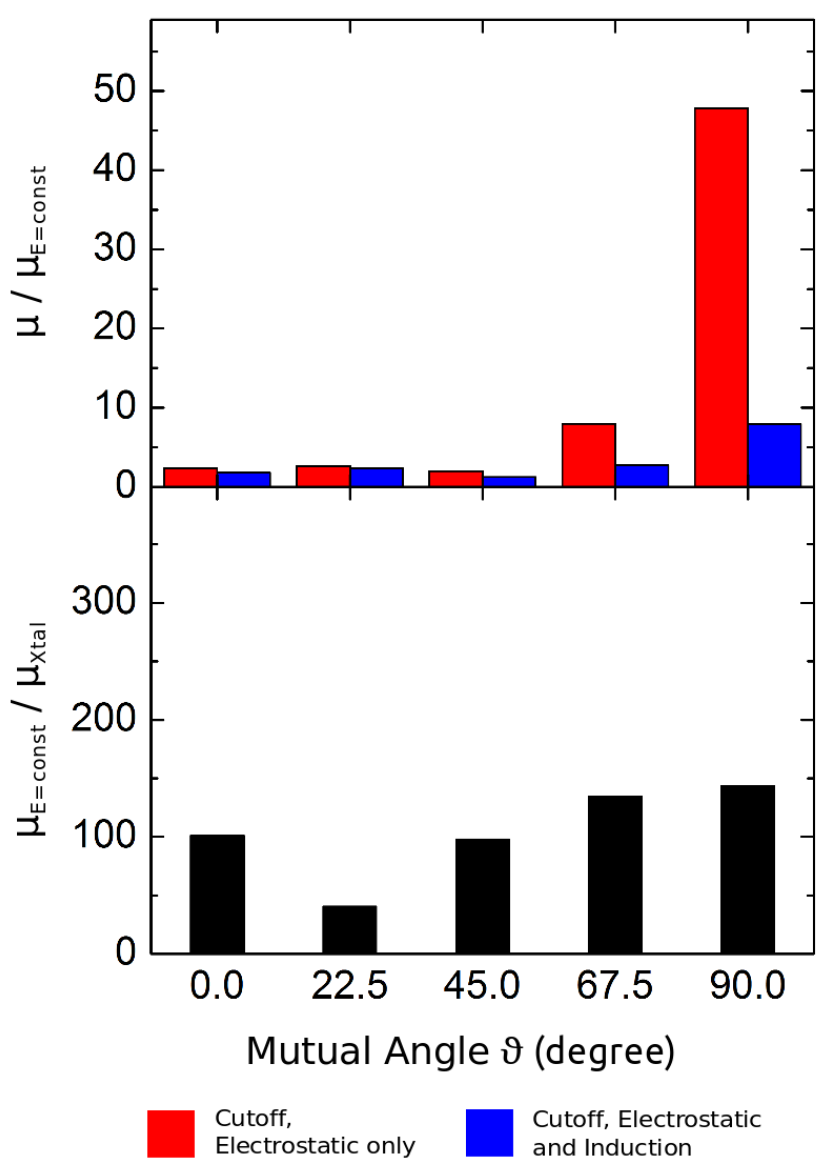

FIG. 12: Standard deviations of mobility ratios for $4 \times 40 \mathrm{~nm}^{2}$ stripes. $\mu_{X \text { tal }}$ refers to the mobility of ideal crystal structure ${ }^{1}$, $\mu_{E=\text { const }}$ relates to the mobility of the molecular dynamics molecular assembly without energetic disorder and $\mu$ is the mobilty of the molecular dynamics molecular assembly including energetic disorder considering different cases.

allow charge carriers to cross at reduced energy cost.

Figures $13 \mathrm{c}$ and $\mathrm{d}$ show accumulated occupation times of charge carriers during $1 \mathrm{~s}$ of charge transport. The orientation of the applied electric field $F$ indicates the direction of charge transport. Dark blue areas relate to regions where no charge transport happens. Yellow and red areas refer to molecules where charge carriers are blocked from traveling. Bright blue areas are regions where good charge transport occurs. This is mainly the case within grains. We observe that charge transport is impeded by large barriers, at regions where three grains join each other and at voids. 

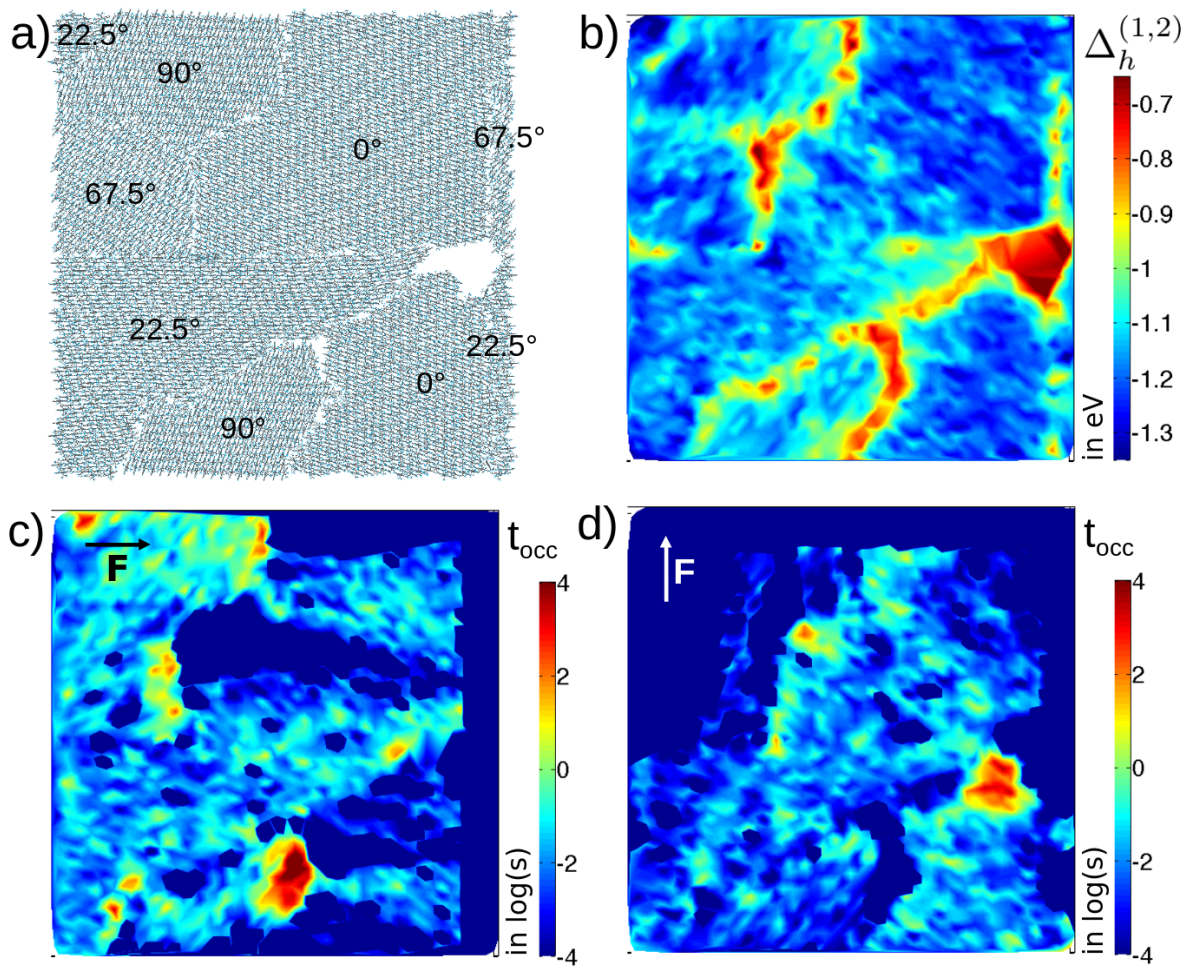

FIG. 13: Charge transport in multi-grain structures of TIPS-P. a) Equilibrated molecular dynamics structure, b) energy surface calculated including contributions of electrostatics and induction, c) and d) accumulated occupation times during $1 \mathrm{~s}$ of charge transport simulations. The orientation of the electric field $F$ indicates the direction of charge transport.

\section{Appendix H: Alternative Representation of Transport Data}

In this section, mobility ratios are flipped compared to the main paper. In this case, we do not observe a clear trend towards larger angles. In the previous representation of Figure 3 (see main text) the average was dominated by few, large mobility reductions. The effect of those outliers are less pronounced when inverting the mobility ratios (see Figure 15). In our PCBM multi-adduct paper (reference ${ }^{35}$ ) we used the represenation of Figures 2 and 3.

Which version would you consider as the fairest representation? 

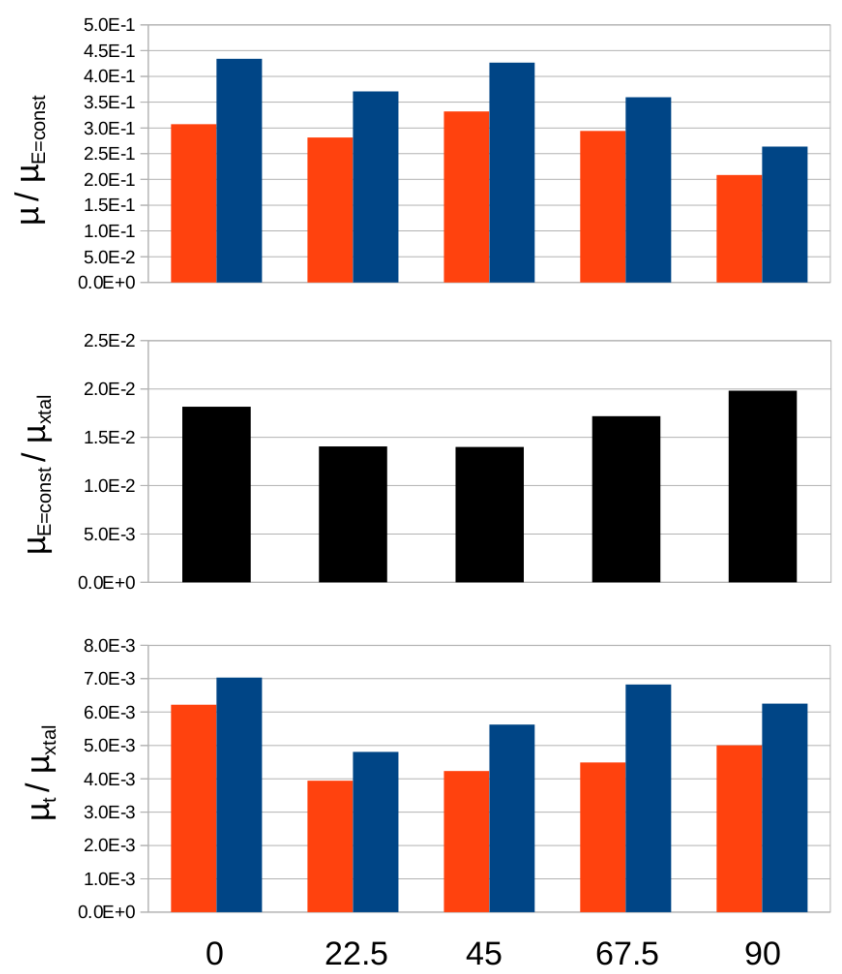

FIG. 14: Average mobility ratios for large $40 \times 40 \mathrm{~nm}^{3}$ structures. Mobility ratios are flipped compared to Figure 2.
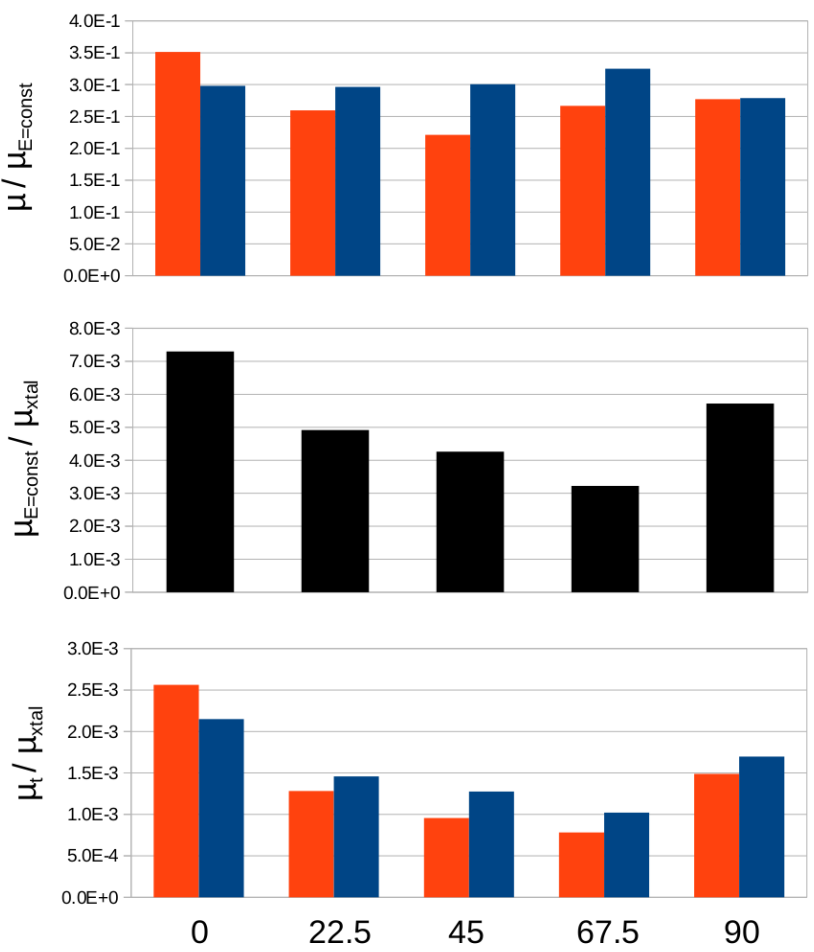

FIG. 15: Average mobility ratios for large $4 \times 40 \mathrm{~nm}^{3}$ structures. Mobility ratios are flipped compared to Figure 3 . 